\title{
CORRIGENDUM
}

\section{Demon-like algorithmic quantum cooling and its realization with quantum optics}

Jin-Shi Xu, Man-Hong Yung, Xiao-Ye Xu, Sergio Boixo, Zheng-Wei Zhou, Chuan-Feng Li, Alán Aspuru-Guzik \& Guang-Can Guo

Nature Photon. 8, 113-118 (2014); published online 19 January 2014; corrected after print 5 February 2014

In the version of this Letter originally published online and in print, the affiliation of Sergio Boixo was incorrectly given. He is affiliated with the Department of Chemistry and Chemical Biology, Harvard University, Cambridge, Massachusetts 02138, USA. This has now been corrected in the HTML and PDF versions of this Letter. 